\title{
Quantum-well-laser mirror degradation investigated by microprobe optical spectroscopy
}

C. Corvasce, Vincenzo Spagnolo, Gaetano Scamarcio, M. Lugara, F. Adduci, et al.

C. Corvasce, Vincenzo Spagnolo, Gaetano Scamarcio, M. Lugara, F. Adduci, Michele Ferrara, Michele Sibilano, Sergio Pellegrino, Massimo del Giudice, M. G. Re, "Quantum-well-laser mirror degradation investigated by microprobe optical spectroscopy," Proc. SPIE 2648, International Conference on Optical Diagnostics of Materials and Devices for Opto-, Micro-, and Quantum Electronics, (3 November 1995); doi: 10.1117/12.226195 Devices for Opto-, Micro-, and Quantum Electronics, 1995, Kiev, Ukraine 
Quantum-Well-Laser mirror degradation investigated by micro-probe optical spectroscopy

C.Corvasce, V.Spagnolo, $\left({ }^{*}\right)$ G.Scamarcio, M.Lugarà, F.Adduci, M.Ferrara, M.Sibilano

Dipartimento di Fisica, Università di Bari, I-70126 Bari, Italy

S.Pellegrino, M.Del Giudice, M.G.Re

Alcatel Telettra, Vimercate-Milano, Italy

\begin{abstract}
A study of facet degradation of InGaAs quantum well lasers is reported. We tune up a Raman and photoluminescence micro-probe technique for determining the crystal structure and the temperature profile of the cladding layer, in steps of $\sim 1 \mu \mathrm{m}$, with a temperature resolution better than $1^{\circ} \mathrm{K}$. The cladding layer composition and cross-section temperature profile have been monitored during operation. A clear correlation between the facet degradation and the type of protective coating is found.
\end{abstract}

Keywords: diode laser, catastrophic optical damage (COD), Raman micro-probe spectroscopy, photoluminescence micro-probe spectroscopy

\title{
1. INTRODUCTION
}

Recently, quantum well lasers have attracted an increasing attention as light source in printers, optical disk memories and optical communication systems. The recent advances in growth techniques have greatly improved the performance and the reliability of quantum well laser diodes. The lifetime is no longer limited by failures in bulk semiconductor, but mainly by facet degradation caused by surface defects that enhance non radiative decay rates and lead to high local heating. ${ }^{1-3}$ Thus, to understand and characterize these thermal effects is fundamental for the development of long lifetime and high stability diode lasers.

In previous works, Raman spectroscopy ${ }^{4,5}$ and reflectance modulation techniques ${ }^{6}$ have been used to study the facet temperature behaviour. Recently a photoluminescence (PL) micro-probe technique has been used to measure the lateral temperature profile of GaAs-based single stripe and broad-area diode lasers. ${ }^{7}$ In this work, we report a Raman and PL micro-probe technique allowing us to directly analyse the local heating and the crystal structure of the cladding layers and to determine their temperature profile perpendicularly to the cavity axis. Different mirror coatings have been compared. We found a clear correlation between facet deterioration and the type of protective coating.

\section{EXPERIMENTAL}

The investigated samples consist of $\mathrm{In}_{0.22} \mathrm{Ga}_{0.78}$ As single quantum well lasers. The lasers have been growth by MOCVD on $\mathrm{n}$-doped (001) GaAs substrate. AlGaAs and InGaP have been used as cladding layer, as reported in table I. 
Table I- Diode lasers structure

\begin{tabular}{|l|c|c|}
\hline & $\mathrm{AlGaAs}$-based laser & InGaP-based laser \\
\hline buffer and substrate & $\mathrm{GaAs}$ n-doped & GaAs n-doped \\
\hline cladding layer & $\mathrm{Al}_{0.5} \mathrm{Ga}_{0.5} \mathrm{As} 1.4 \mathrm{~mm}$ & $\mathrm{InGaP} 2 \mathrm{~mm}$ \\
\hline guide layer & $\mathrm{Al}_{0.2} \mathrm{Ga}_{0.8} \mathrm{As} 550 \AA$ & $\mathrm{InGaAsP} 1000 \AA$ \\
\hline active layer & $\mathrm{GaAs} 60 \AA$ & $\mathrm{In}_{0.22} \mathrm{Ga}_{0.78} \mathrm{As} 70 \AA$ \\
\hline contact layer & $\mathrm{GaAs}$-doped $2000 \AA$ & $\mathrm{GaAs}$ p-doped $2000 \AA$ \\
\hline
\end{tabular}

Uncoated InGaP-based laser, AlGaAs-based laser with facets cleaved in air and then coated with $\mathrm{Al}_{2} \mathrm{O}_{3}$ and $\mathrm{AlGaAs}$-based laser with facets cleaved in air, deoxidized and then coated with $\mathrm{Al}_{2} \mathrm{O}_{3}$ have been investigated. Raman scattering and photoluminescence (PL) have been excited at room temperature in a backscattering geometry by focusing the $5145 \AA$ line of an $\mathrm{Ar}^{+}$ laser directly onto the active region. Typical spots of $\sim 1 \mu \mathrm{m}$ are obtained, smaller than the cladding layer width of the investigated sample. The spectra were measured by using a Jobin-Yvon T64000 triple spectrometer with focal length of $0.64 \mathrm{~m}$. The signal was detected by an $\mathrm{N}_{2}$-cooled CCD system. In order to avoid sample heating effects, the incident power density was kept below $10^{2} \mathrm{~W} / \mathrm{cm}^{2}$.

\section{RESULTS AND DISCUSSION}

Figure 1 shows $\mathrm{PL}$ spectra generated in the $\mathrm{Al}_{0.5} \mathrm{Ga}_{0.5} \mathrm{As}$ cladding layer at different diode currents. The shift of peak position of the PL as a function of the temperature has been calibrated by probing $\mathrm{Al}_{0.5} \mathrm{Ga}_{0.5} \mathrm{As}$ and InGaP bulk samples with the same $\mathrm{n}$-doping concentration of the cladding layer, by varying the sample temperature with a heater. Thus, from the shift of the peak position of the PL band, we are able to detect changes in the cladding layer temperature smaller than $1^{\circ} \mathrm{K}$.

By increasing the diode current up to the catastrophic optical damage (COD), a clear correlation has been found between the output power and the facet temperature. In figure $2 a$ the output power and the cladding layer temperature shift are shown as a function of the diode current $I_{d}$ for an AlGaAs-based diode laser with non deoxidized facets. Both the temperature and the output power increase at increasing $I_{d}$ up to the COD. Similar results are obtained for the uncoated InGaP-based lasers, except for the output power which saturate at $I_{d}=180 \mathrm{~mA}$ and then decreases by further increasing $I_{d}$, as shown in figure $2 b$.

Micro-Raman analysis allows us to detect any change in the crystal structure of the cladding layer. In figure $3 a$ and $3 b$ Raman spectra recorded before and after the COD for a coated AlGaAs-based and an uncoated InGaP-based sample, respectively, are reported. No significant deterioration after the COD is observed for the coated sample. On the contrary, a drastic change in the Raman spectrum is observed for the uncoated structure. After the COD, the InGaP related band almost disappears, whereas the InGaAs one rises. This is due to Phosphorus (P) desorption induced by the huge rise of the temperature at the COD. Thus the Raman analysis suggests that while in the uncoated InGaP-based structure the COD is due to a P desorption, in the AlGaAs-based lasers the COD is probably related to a crystal deterioration of the inner layers of the diode, i.e. guide and active layers. 
In figure 4 the temperature profile of the cladding layer of a coated AlGaAs-based laser with deoxidized facets, obtained at different diode current, is shown. The layer temperature rapidly decreases at increasing the distance $(\Delta a)$ from the active region, demonstrating that most of the heating damages are suffered only by a small region of the sample, centred on the active layer.

The comparison between the temperature increases of the cladding layer, close to the active region, in the laser diode with cleaved and coated facets and in the laser with cleaved, deoxidized and then coated facets, clearly shows that in the latter the facets stay cooler; for example at $\mathrm{I}_{\mathrm{d}}=150 \mathrm{~mA}$ the cladding layer temperature for deoxidized and non-deoxidized facets is $\Delta \mathrm{T}=16^{\circ} \mathrm{K}$ and $\Delta \mathrm{T}=32^{\circ} \mathrm{K}$, respectively (see figure $2 \mathrm{a}$ and 4 ). This can be ascribed to a reduced incorporation of defects in the mirror layers.

\section{CONCLUSIONS}

In conclusion, we have demonstrated how the Raman and PL micro-probe technique can be used as a powerful tool in determining the crystal structure and temperature profile of the cladding layer of quantum well lasers, during operation, with a spatial and a temperature resolution of $\sim 1 \mu \mathrm{m}$ and better than $1^{\circ} \mathrm{K}$, respectively. A clear correlation is found between the output power and the cladding layer temperature up to COD. Raman analysis suggests that in uncoated InGaP-based diodes the COD is due to $\mathrm{P}$ desorption induced by the high facet temperature and in coated AlGaAs-based lasers is probably related to crystal deterioration of the guide and active layer, while the cladding layer structure is nearly undamaged. The comparison between AlGaAs-based laser with non-deoxidized and deoxidized facets clearly shows that the latter process leads to a reduced incorporation of defects in the mirror layers.

\section{ACKNOWLEDGEMENTS}

This work has been partially supported by Italian CNR (Research National Council).

\section{REFERENCES}

$\left(^{*}\right)$ Present address: AT\&T, Bell Laboratories, Murray Hill, NJ-07974, U.S.A..

1. T.Yuasa, M.Ogawa, K.Endo, H.Yonezu, "Degradation of (AlGa)As DH lasers due to facet oxidation," Appl. Phys. Lett. Vol. 32, issue 2, pp. 119-121, January 1978.

2. W.C.Tang, H.J.Rosen, P.Buchmann, P.Vettiger, D.Webb, "Probe beam heating of AlGaAs single-quantum-well laser facets in Raman microprobe spectroscopy," J. Appl. Phys. Vol. 62, issue 11, pp. 5930-5932, December 1990.

3. A. Moser, "Thermodynamics of facet damage in cleaved AlGaAs lasers," Appl. Phys. Lett. Vol.59, issue 5, pp. 522-524, July 1991.

4. S.Todoroki, M.Sawai, K.Aiki "Temperature distribution along the striped active region in high-power GaAlAs visible lasers," J. Appl. Phys. Vol. 58, issue 3, pp. 1124-1128, August 1985.

5. H.Brugger, P.W.Epperlein, "Mapping of local temperatures on mirrors of GaAl/AlGaAs laser diodes," Appl. Phys. Lett. Vol. 56, pp. 1049, 1990.

6. P.W.Epperlein, G.L.Bona, P.Roentgen, "Local mirror temperatures of red-emitting (Al)GaInP quantum-well laser diodes by Raman scattering and reflectance modulation measurements," Appl. Phys. Lett. Vol. 60, issue 6, pp. 680-682, February 1992.

7. D.C.Hall, L.Goldberg, D.Mehuys, "Technique for lateral temperature profiling in optoelectronic devices using a photoluminescence microprobe," Appl. Phys. Lett. Vol. 61, issue 4, pp. 384-386, July 1992. 


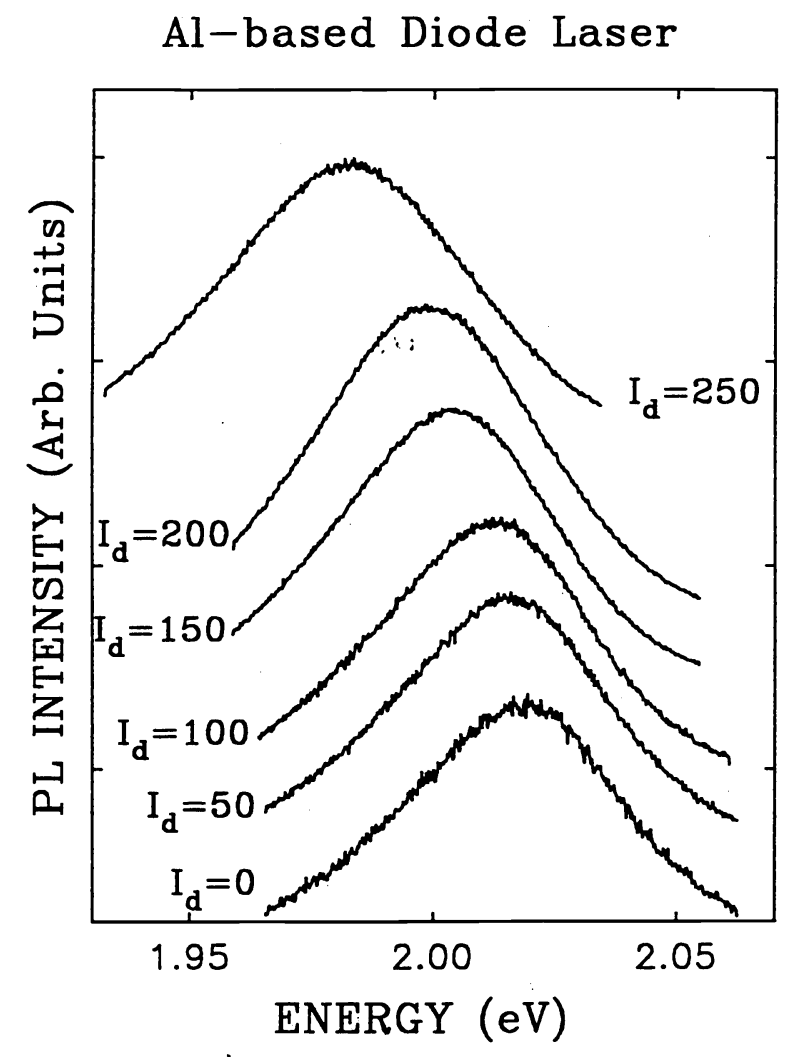

Figure.1. PL spectra generated in the $\mathrm{Al}_{0.5} \mathrm{Ga}_{0.5} \mathrm{As}$ cladding layer at different diode currents $\mathrm{I}_{\mathrm{d}}$

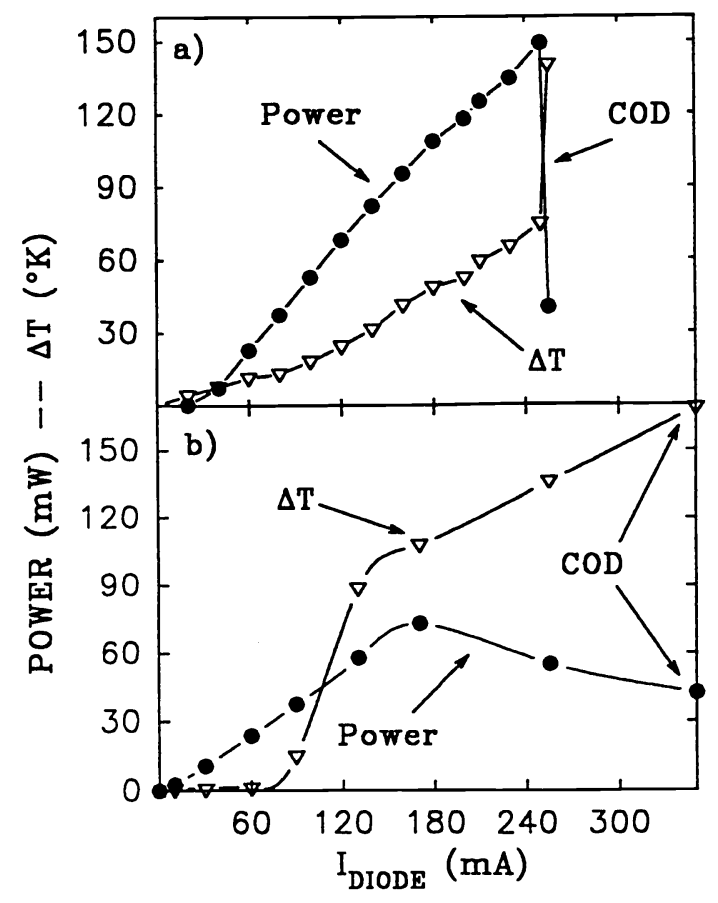

Figure.2. Output power and cladding layer temperature shift as a function of diode current $I_{d}$ for coated AlGaAs-based with non deoxidized facets (a) and uncoated InGaP-based (b) diode lasers. 


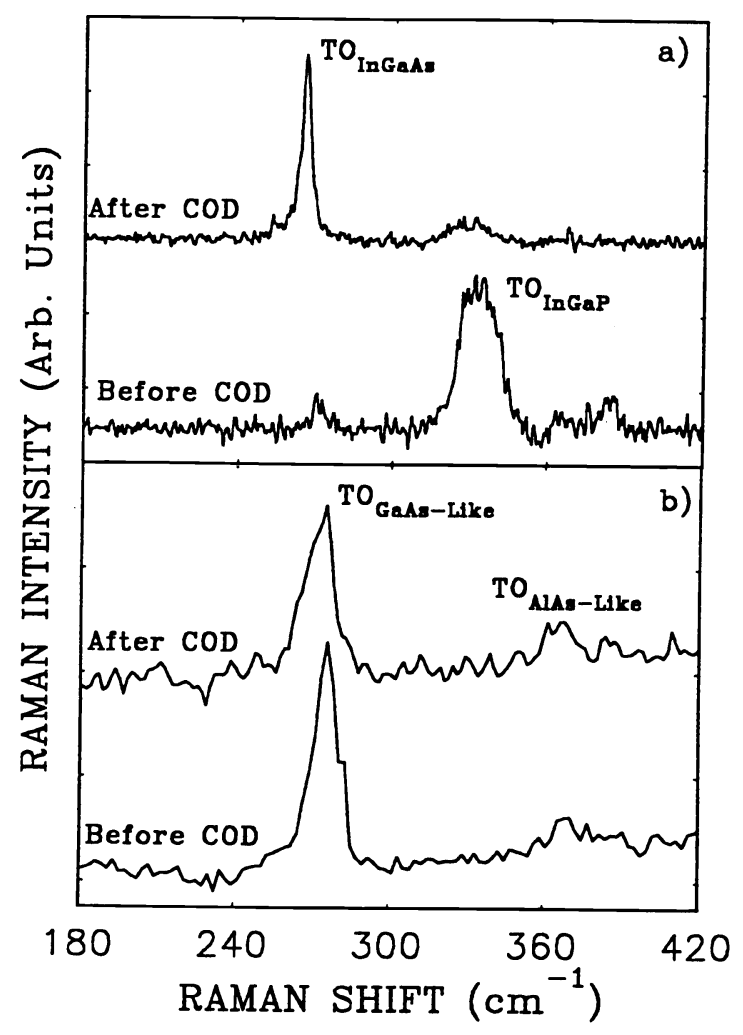

Figure.3. Raman spectra obtained before and after the COD for coated AlGaAs-based with non deoxidized facets (a) and uncoated InGaP-based (b) diode lasers.

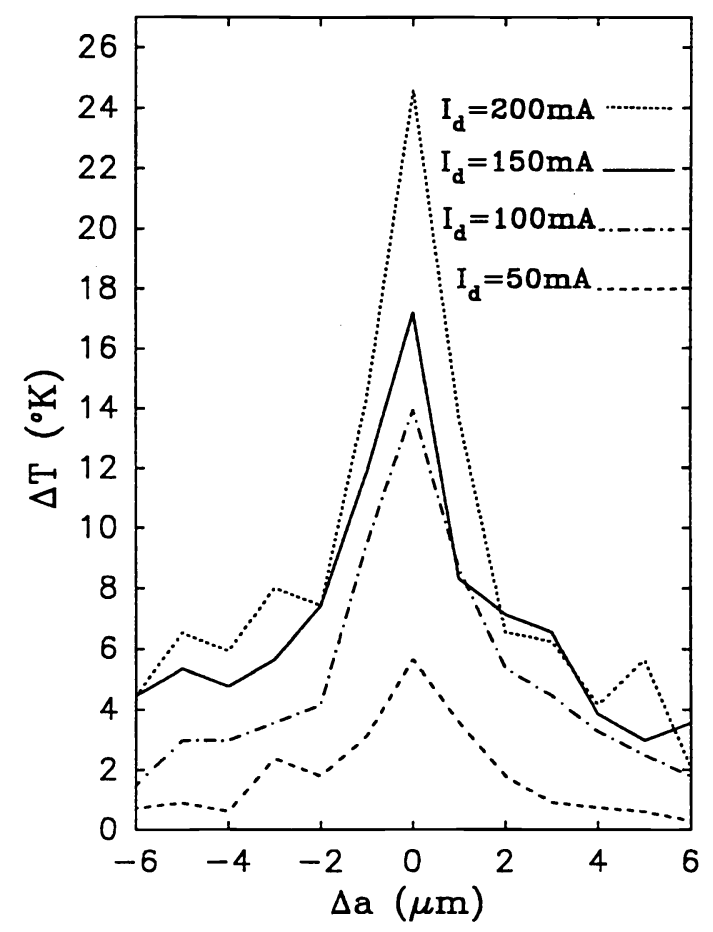

Figure.4. Temperature profile obtained at different diode currents $I_{d}$ for coated AlGaAs-based with deoxidized facets. 